\title{
Trajectories in Multiple Group Coordination: A Field Study of Hospital Operating Suites
}

\author{
Yuqing Ren, Sara, Kiesler, Susan Fussell, Peter Scupelli \\ Human-Computer Interaction Institute \\ Carnegie Mellon University \\ \{yren, kiesler, sfussell,pgs\}@andrew.cmu.edu
}

\begin{abstract}
Hospital surgery environments are dynamic and high risk. They require coordination across multiple groups whose incentives, cultures, and routines can conflict. In this paper, we describe a field study of multi-group coordination in the operating room (OR) environment. We studied work trajectories from the perspective of each group involved. Coordination breakdowns at group boundaries involving patients, doctors, nurses, and other staff led to local problem solving, but also to delays and interpersonal tension and conflict across groups. We conclude with design recommendations for multiple group coordination support that would promote trajectory awareness and learning at the organizational level
\end{abstract}

\section{Introduction}

Surgical procedures require the coordinated efforts of surgeons, nurses, anesthesiologists, and other support personnel working under stress and time pressure [10]. Large investments have been made in sophisticated equipment for patient-monitoring and displays. Computer-based scheduling systems based on operations research techniques have been installed to increase efficiency and reduce staff turnover [6]. Attention has turned more recently to understanding coordination needs of medical personnel and how technology can support coordination in the medical setting. Bardram [2] described how coordinating activities unfold over time in a surgical department. Reddy and Dourish [22] examined information exchange in a surgical intensive care unit and the role of temporal rhythms as a source for interpreting and coordinating work. Moss and Xiao [17], Seagull et al., [23], and Xiao et al. [29] studied communication patterns and coordination practices in trauma centers; they identified problems that complicate coordination such as optimistic schedules, temporal constraints, and status differences in the organizational hierarchy.

With some exceptions [19], [25], most research on coordination in hospitals and in other safety-critical organizations examines processes from the point of view of one role or collocated group or unit - such as workers in an emergency call center, a traffic control room, or an Intensive Care Unit (e.g., [2], [9], [22]). This line of research has greatly deepened our understanding of coordination processes, information exchange, and how workers switch attention between their own and collaborative tasks [9]. It also identifies common problems that workers face in hospitals such as distractions, conflicts, obstacles requiring workarounds [12], and political allocation of resources.

The current paper differs from prior work in that our goal was to address coordination across multiple groups operating in and surrounding hospital OR units, as seen from the point of view and activities of each group involved, including patients. We try to answer several questions. What are the key challenges or factors associated with multiple group coordination? How do geographic and organizational boundaries complicate operation coordination practices? How can current technologies be improved or modified to facilitate multiple group coordination? By observing coordination practice across multiple group boundaries, we hope to gain a better understanding of the challenges that computer support must meet to support collaborative work in the OR environment.

We conducted an in-depth field study of OR coordination practices in a Mid-Atlantic hospital. We used the concept of trajectory to focus our observations on workflow and coordination across groups. Trajectory is defined as the sequence of activities and paths through which people, resources, and groups move [24]. Patients, doctors, nurses, and other personnel each move through a trajectory through tasks and time. Resources such as operating rooms and equipment also have expected trajectories while being 
utilized case after case to serve different patients. By examining sources and consequences of coordination breakdowns around OR trajectories, we identified two critical factors in complex, dynamic coordination trajectory awareness and organizational learning, and proposed concrete technical and social solutions to improve multiple group coordination.

\section{Research setting and method}

Our research site was a major hospital complex located in an urban setting in the Mid-Atlantic region. Its specialties include cardiothoracic surgery, organ transplantation, critical care and trauma services, and neurosurgery. It is also designated as a level I regional trauma center. We conducted observations in the operating room (OR) units and all the groups that participate in surgical services. The units that we observed are distributed in two hospital buildings that are located adjacent to one another. The OR units in the two buildings have their own pre-operative and post-operative holding areas and share personnel and resources. In this situation, coordination is required not only among multiple professional groups, units, and roles within each OR unit but across the two hospital buildings. The OR units are the biggest revenue generator in the hospital. They have 43 operating rooms located in the two hospital buildings that we refer as POR and MOR. On a regular workday, 40 to 50 surgeries are scheduled in POR and 30 to 40 surgeries are scheduled in MOR.

We collected data using observations, interviews, and the Critical Incident Technique [7]. We used the ethnographic approach of taking every group's perspective into account, including patients and workers who transport patients from place to place. We spent 195 hours between February and June of 2005 observing at the OR desk, anesthesia whiteboard, and work areas of other groups, and shadowing charge nurses and charge anesthesiologists. We spent the first two months getting familiar with the environment and understanding the general procedures. We conducted interviews during observations to clarify or amplify the observations. In-depth interviews were conducted with 2 surgeons, 7 anesthesiologists, 6 nurses, and 5 others (e.g., secretaries, unit coordinators, and escorts), and lasted between 15 and 30 minutes. The authors took detailed notes during field visits. Notes from the early stage of the project were compared and discussed among the authors to assure quality. All of the notes were coded and analyzed using Nvivo [21].

\section{OR organization}

OR organization is dominated by the primacy of the schedule [2]. In the organization we studied, the schedule follows a daily block system. OR blocks are groups of rooms assigned to different services such as cardiac, neuro, thoracic surgery, and so on, as well as to individual surgeons. To optimize OR utilization, 48 hours prior to the operating day, unbooked OR times are made available for other surgeons or services. The schedule allows for a 30-minute break between scheduled cases to provide for anesthesia and room turnover time. The schedule is variable across time. Schedulers specify surgery duration as whatever is requested by surgeons plus an adjustment based on the surgeon's historical record. Every morning, a daily operating room schedule is printed with patient, procedure, and physician information. This schedule is used by all the units that are related to surgery.

\subsection{Multiple groups}

There are normally six groups involved in surgical services: ambulatory, pre-operative holding, operating room nurses, anesthesia team, surgical team, and post anesthesia care. Two other groups that are not primary care providers but key players are housekeeping and patient transportation.

Ambulatory surgery is responsible for interviewing and admitting patients when they first arrive. Nurses and technicians get patients ready for surgery by making sure that they change to hospital gowns, have followed all pre-operative instructions, and all required testing results are included in the patient charts.

Nurses working in the pre-operative holding area (POHA) monitor patients' vitals and double check the patient charts to make sure that all required information is included. These workers also are responsible for notifying the OR desk, and anesthesia and surgical teams when patients arrive.

When a patient arrives in an operating room, a circulating nurse greets the patient, records critical times and OR charges, and assists the surgical team in positioning the patient and finding equipment. Scrub technicians are responsible for providing and keeping track of all sterile equipments. An OR charge nurse, who normally sits at the OR information desk, is responsible for running $\mathrm{OR}$ scheduling and for coordinating activities with other groups.

An anesthesia team usually consists of an anesthesiologist and one or two hands-on providers. These workers may be an anesthesiology resident or a nurse anesthetist, who sometimes supervises a student 
anesthetist. Anesthesia teams are responsible for visiting patients in POHA (the pre-operative holding area), transporting patients from POHA to OR, anesthetizing patients, monitoring patients during surgery, waking up patients and transporting them to post-anesthesia recovery.

When a patient is transported to the post anesthesia care unit (PACU), PACU nurses take a report from the anesthesia team, settle the patient in bed, and closely monitor patient pain and vital signs until the patient is stable enough to go to a floor or go back to same day post-operative in ambulatory surgery to be discharged.

Groups follow different work trajectories that intersect with and join with other groups at different times. OR practices involve three key trajectories: patient trajectory, staff trajectory, and resource trajectory. A typical patient trajectory is arriving at the hospital, getting ready in POHA, moving to a room to receive surgery, staying at PACU, and being discharged. An example of a staff trajectory is the surgeon trajectory. It may involve surgical duties in the morning and teaching or clinical duties in the afternoon, and nonhospital duties such as working on financial reports or working on an online schedule in the evening. Examples of resource trajectories are the operating room and equipment carts for surgeries. The operating room trajectory is normally scheduled for a series of daily surgeries that may vary in their time and usage. Different trajectories need to interweave and intersect at specific times to provide timely, safe, and high-quality patient care.

\subsection{An overview of the patient trajectory}

We illustrate the complexity of managing and coordinating multiple trajectories using a patient trajectory as the focal point. The hospital treats both inpatients and outpatients, but we chose to study outpatient services because we had the opportunity to observe the full course of most outpatient cases. Many inpatients already had a history of being treated in the

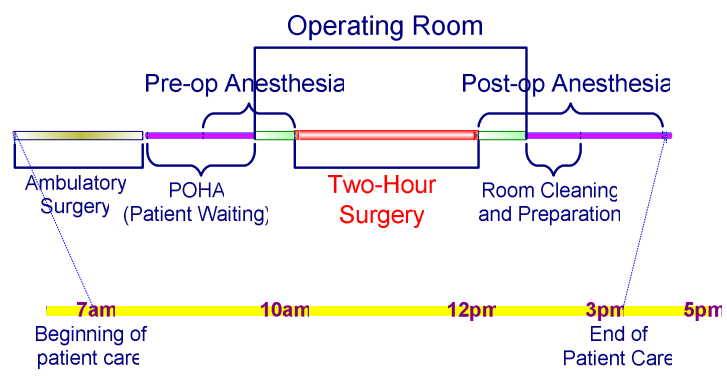

Figure 1. An example patient trajectory hospital or operating rooms well before they entered our zone of observation. An outpatient trajectory consists of three stages: pre-operative preparation, operating, and post-operative recovery.

Suppose a patient is scheduled to have surgery between 10am and noon. The patient will be instructed to arrive 2.5 or 3 hours before the scheduled surgery. As shown in Figure 1, the patient first arrives at the ambulatory surgery unit located in building $M$ where he or she is admitted and interviewed to get ready for surgery. The patient is then place in a wheelchair or on a gurney and transported by escorts to a pre-operative holding area located in building $\mathrm{P}$ (or a different floor in building $\mathrm{M}$ ) to meet the anesthesia team. Someone on the team rolls the patient to the designated operating room, also in building $\mathrm{P}$ (or $\mathrm{M}$ ). A member of the surgical team needs to check the identity of the patient before the anesthesia team anesthetizes the patient.

After the patient is anesthetized, the surgical team opens the patient, performs surgery, and closes the surgical procedure. After the surgery, the anesthesia team stops sedation, wakes up the patient, and rolls the patient to the post anesthesia recovery area. At the same time, someone from the anesthesia team meets the next patient in the pre-operative holding area and prepares for the next surgery. Once the patient is out of the room, housekeeping cleans the room, and the circulating nurse and a scrub technician restore the room with proper trays and equipment. The room is ready for the next patient.

Three types of interdependency are present and intermingled [19] in the trajectories. There is sequential interdependence, for example, housekeeping cannot clean the room until the patient is physically out of the room, and the next patient cannot be rolled in until the room is properly cleaned and equipped. There is reciprocal interdependence in the way groups must exchange information or complete actions for another group to proceed with their own tasks. For instance, the anesthesia team needs to wait for the surgical team to ID the patient before putting the patient to sleep, and the surgical team cannot start operating until the patient is anesthetized. There is also pooled interdependency, which means dependence on shared resources or behavior. Many activities in OR share common resources such as personnel, rooms, and equipment. When these interdependencies are not synchronized, coordination breakdowns may occur.

\section{Coordination breakdowns}

In previous studies, breakdowns in the hospital environment mainly concerned deviations from the 
formal procedures or errors in patient care, such as failures to monitor patient status during surgery (e.g. [10], [25]). Our focus is breakdowns observed in the coordination and workflow of multiple trajectories. We define coordination breakdowns as changes in, or barriers to, trajectories, to which people and resources must adjust. Breakdowns are thus triggers for essential changes in coordination. Most breakdowns that we observed occurred at group boundaries, especially around the time when patients moved in and out of operating rooms.

\subsection{Sources of coordination breakdowns}

OR scheduling and coordination would not be a major challenge if events always ran as scheduled. Instead, breakdowns occur as an inherent part of medical care and OR practices. Emergency cases consume resources that are originally assigned to elective cases. Unexpected changes in patient condition turn an elective case into an emergency. OR staff call off due to personal problems. Doctors get stuck with patient care outside of the hospital and arrive late. Following [15], we categorize sources of coordination breakdowns as internal and external interruptions. We emphasize internal interruptions as design opportunities because most external interruptions such as emergency and transplant cases are completely stochastic and impossible to predict or control.

\subsection{Interruptions from patients}

There are three sources of internal interruptions from patients: transmittal of incomplete information, changes in their physical condition, and their disruptive behavior. Our data suggest that most behavioral interruptions from patients are unintentional and stem from patients' ignorance of medical practices, or "poor medical management" on the hospital side. A common reason for patient delay is incompleteness of patient documents such as patient charts, consent forms, or history and physical forms.

\footnotetext{
"Patients come from all over the country, and their documents failed to be faxed ahead of time ... One difficulty in obtaining patient information is that a lot of places don't send you anything without a medical release. You call the lab and they can't give it to you until the morning when the patient comes in and gives a medical release (because of HIPPA).” [Ambulatory Nursing]
}

Another common interruption happens when patients' conditions change - either for the worse, requiring emergent surgery (e.g., internal bleeding), or for the better, requiring cancellation of the surgery.
Sometimes patients failed to follow diet or medication instructions (e.g., patients get dehydrated or eat 24 hours before the surgery). We also observed two incidents in which the patients refused to sign consent forms or even refused surgery and the cases had to be cancelled at the last minute.

"She (the patient) is refusing to come down. Nobody has talked to her. Can you send somebody up? ... She has had too much today and she is not coming." [OR Nursing]

In another case, the patient was multi-tasking. His surgery was scheduled at 10:45am and he made an appointment at his doctor's office at 10am. After talking with pre-op nurses, the patient insisted on keeping his plan.

"Once the patient signed in, he is our responsibility. He was supposed to wait and we took him to bedside. The patient walked out anyway. His appointment is at 10am and his surgery is scheduled at 10:45am. He said the office is aware. ... Eventually we sent him over, but OR had to change their schedule to not lose OR time." [Ambulatory Nursing]

\subsection{Interruptions from surgeons}

Surgeons' busy schedules and complex trajectories create the second major source of interruptions in the OR unit. As compared with anesthesiologists and nurses who work regularly in operating rooms, surgeons attend only when they have cases scheduled. They often have obligations elsewhere such as teaching and seeing patients in their offices or operating in other hospitals before or after their scheduled surgeries. When these activities run over time, they are not available to do their surgery at scheduled times.

"That was a surgeon issue. Surgeons work at different hospitals and they may be working overnight at another hospital and not available to start his case here at [hospital name omitted]. For this case, all we were told was the surgeon was not available. We don't ask about the details. It could be personal reasons as well." [Ambulatory Nursing]

In a teaching institution like the one we studied, it is a common practice for surgeons to do the critical part of a surgery and have their residents or fellows do the opening and closing parts. On busy days, a surgeon (and his surgical team) may have two or three surgeries in different rooms ongoing at the same time. The surgeons "flip and flop" between the rooms. Although this practice enables them to serve more patients, it increases the probability of a case running late.

In one case that we observed, the surgeon put a room on hold so that she would not lose it. In many other circumstances, it is more common for surgeons to 
request an additional room (mostly due to add-on cases) or a better-equipped room.

"Dr. [last name omitted] has sent for patient in 7 but we can't go back until she is ready. She has another case in 8 and she can't leave 8 yet. ... We would have to wait 45 minutes to one hour to go back to the room. The patient is somewhere in the hospital. The surgeon doesn't want to lose the room as it gets closer to 3pm." [OR Nursing]

\subsection{Interruptions from anesthesia and nursing}

Hospital personnel call all of the nurses and technicians who provide care to surgical patients "Nursing." From the Nursing perspective, many interruptions are caused by frequent staff turnover and new nurses' lack of experience, as illustrated in the following comments:

"Cases get delayed because of this (lack of experience). This is like sending soldiers to Iraq with only a sleeping bag. ... We need to have some ways to assess this to make sure that qualified people are assigned to rooms. You don't assign untrained nurses to orthopedics." [Surgical]

In this case, we observed that a nurse involved in a complex surgery had been with the hospital for less than a year and "was not fine-tuned and did not know the surgeon's routines very well." This phenomenon is not unique among OR nurses. We observed a similar incident in ambulatory surgery where an inexperienced call nurse mistakenly told an add-on patient to come to the hospital though the case was not scheduled.

Similar issues may affect the anesthetic team. The anesthesia team's primary responsibilities are patient airway and sedation. Patients with special conditions may react differently to anesthetic procedures and medications. Therefore, anesthesiologists and their hands-on providers need "to anticipate problems and plan ahead." With difficult cases in more than one room, anesthesiologists need to triage and, if necessary, to seek for help from coworkers. Failing to do either can result in delays or mistakes.

Another major source of interruption is inadequate staffing, particularly of nonmedical workers such as escorts and housekeeping staff. Although these two groups do not serve patients directly as doctors and nurses do, they have a significant impact on the OR schedule. Cases cannot start until rooms are cleaned and patients are transported to the room. Understaffing in these two groups results in bottlenecks and delays on a regular basis, so much that people constantly complain about it. Here are two examples.

"In a short period of time, POR called for five patients and MOR called for three patients. There are only two escorts who transport patients from same day to here and also between MOR and same day, which is one level apart. As a result, this case got delayed, which was frustrating. This surgeon was always on time." [Pre-op Nursing]

"Room turnover is a big issue.... Most times people blame anesthesia for delays. But a lot of times rooms are not ready because of housekeeping or nurses not having equipment in the room." "Why is room turnover not faster here?" "This hospital is too big. They don't have enough manpower, like housekeeping. There is a housekeeping team assigned to work in OR. They are too busy." [Anesthesia]

\subsection{Interruptions across groups}

Groups interrupt each other. Key decision makers such as charge nurse and charge anesthesiologists are constantly interrupted with requests for information and unexpected events that require adjustments in the schedule. As surgeons multitask and juggle their responsibilities, they not only interfere with the work flow in operating rooms, but also with pre- and post-op patient care. For instance, it is required that someone from the surgical team talks to the patient and family before and after the surgery. Surgeons perceive these as peripheral activities and perform these duties whenever they are not tied up with other more important responsibilities. We observed many cases in which "higher-profile" workers interrupted "lower-profile" workers. Doctors and anesthesiologists interrupted nurses and nurses interrupted technicians.

\section{Consequences of breakdowns}

People working in the operating suites need to adjust to coordination breakdowns when they occur. Cases need to be rearranged, priorities need to be reassessed, and staff may be reallocated. When groups fail to act collaboratively to adjust to breakdowns, negative consequences can happen. In this section, we discuss consequences of interruptions and coordination breakdowns to organizations as well as individuals affiliated with the organizations. To assure confidentiality, we deliberately exclude individual titles and use "he" consistently no matter whether a female or male was involved in the following events.

\subsection{Organizational consequences}

From an organizational perspective, interruptions can have both positive and negative consequences. On the positive side, interruptions instigate coordination in real time, which can be an efficient "market driven" method of allocating people's attention and resources. On the negative side, interruptions may cause 
breakdowns that cannot be easily resolved and can lead to delays and reduced profit margins. Also, interpersonal tension and conflict arise when groups must continually attend to coordination when, at the same time, workload and stress are very high.

5.1.1. Delays. Efficiency and resource utilization are major concerns of the hospital administration. People adjust to coordination breakdowns to solve problems, synchronize activities, and sort out priorities. When people fail to adjust in a timely manner, delays occur. Every time an operating room is put on hold, patients, families, and the whole OR team will be waiting. Delays thus lead to financial losses and low profit margins. OR is the biggest revenue generator in the hospital that we studied and in many other hospitals as well. Every time an operating room is put on hold, the hospital has to pay workers to sit around and wait instead of taking care of patients. Additional costs are incurred outside of operating rooms. Pre-operative and post-operative charges are either a flat rate across cases, or a flat rate by case categories (e.g., neuro, transplant, etc.). When a patient gets stuck in one of these places and blocks personnel and resources that could be otherwise utilized, the delay significantly boosts costs and reduces the hospital's profit margins.

"It is a stochastic process organized around emotions. It is pointless to model it using the conventional orderly process. We have a $\$ 42$ per minute OR team waiting for a 9-cent escort. This is the kind of disorder built into the system." [Anesthesia]

5.1.2. Intergroup Tension and Conflict. Groups have incentives, temporal cycles, and considerations that are not always aligned with those of other groups [16]. Surgeons have flexible working hours and are motivated to get many cases done. Like surgeons, anesthesiologists have flexible working hours so that they go home early on light days and stay late when necessary. In contrast, OR nurses work on shifts and are paid by an hourly rate. Already struggling with constant turnover and inexperienced nurses, charge nurses strive to get their staff out on time and to minimize overtime work.

The highly dynamic nature of the environment and constant interruptions to the OR schedule add an extra burden to negotiations. When a patient is delayed or a room is put on hold, groups blame one another especially the party whom they think has contributed to the delay. Surgeons get mad at anesthesia teams or nurses, and anesthesia and nursing teams complain about surgeons' big egos and narrow focus. Everyone gets frustrated with escorts and housekeeping. Some comments are very emotion-intensive, such as "I was spitting bullets today and I chewed him."

"Everybody yells at you. You have to be on your feet all the time and not piss off anybody. You need to keep peace on the floor. I have got [titles omitted] yell at me. If you don't keep the peace, you get in trouble." [Ambulatory Nursing]

"[The biggest challenge is] resistance. People put up resistance to change. We are good within our department. Outside of our department, people don't want to be of help. I make phone calls and sometimes it is unpleasant phone conversations. People say, no, we are not going to do that. It is the patient that we are trying to help. People say, we are not staffed for that and we can't work with that volume." [Ambulatory Nursing]

\subsection{Individual consequences}

From an individual perspective, when a case gets delayed, patients may experience unnecessary waiting or sedation, OR staff may have to work overtime to finish cases that are delayed or bumped during the day, and surgeons may be late for their responsibilities outside of the OR or hospital. Consequently, everyone experiences and suffers from frustration, stress, and fatigue.

5.2.1. Patient. When coordination fails and a case gets delayed, patients may have to wait longer to go to surgery. If an interruption happens after a patient is already sedated, patients may get unnecessary sedation. Because the hospital that we studied is highly prestigious, patients come from all over the country. Although hospital policy gives higher priority to outpatients and patients with special constraints, it has happened that patients needed to come back the next day, which disrupted their families and their personal plans outside of the hospital. Although we did not observe or interview patients directly, they naturally become the foci of conversations.

"It doesn't affect me very much. But it is a waste of time and the poor patient has been waiting for one hour. We have nothing to do, but sit and wait. ... the patient family is waiting. The patient is getting unnecessary anesthesia." [OR Nursing]

5.2.2. Surgeon. Theoretically, surgeons have the biggest clout because of their specialized expertise and prestigious status. One nurse said surgeons are "guests who are invited to a party" that is organized and run by anesthesiologists and OR charge nurses. Surgeons' decisions may have significant impact on other stakeholders. Breakdowns that happen anywhere along 
their trajectories can interrupt their own work plan both within and outside of the operating room.

A typical conversation between surgeons and OR desk or pre-op desk is: where is my patient or is my patient here? When their cases are delayed, surgeons get upset because they will finish fewer cases, may need to stay overtime and work with different nurses as nurses change shifts, and will neglect responsibilities that they are committed to outside of OR.

"It delays your surgery. I have two surgeries scheduled for today. I have office hours (seeing patients in my office). [Someone passed by and said to the surgeon, "You are fast."] They put up every means to delay me." [Surgical]

5.2.3. Anesthesia and nursing. As individuals who work primarily in OR and are in charge of OR scheduling, change anesthesiologists and charge nurses experience the most significant impact from exceptions and interruptions. When delays happen, OR staff stay overtime. They get stressed and frustrated by this aspect of their work. We were told that turnover rates in operating room units have been consistently high in the past decade, nationwide.

"[When a case is delayed,] I send my people to get coffee. [As a result,] the room may not be done at 3pm as it is supposed to be. I would have to pay overtime to have my people stay after $3 \mathrm{pm}$. That would kill you if it was my fault [to put OR on hold]. OR time is $\$ 44$ per minute. We will have to pay people to sit around and wait. Also, it may affect the number of rooms that we can run at $3 \mathrm{pm}$ and we may have to close some rooms." [OR Nursing]

\section{Lessons learned from the field}

\subsection{Importance of trajectory awareness}

Interruptions and coordination breakdowns can happen anytime and anywhere in OR workflow. Often these can be fixed on the spot. However, because of interdependence across groups, small breakdowns within groups can escalate and spill across group boundaries. To minimize these escalations, people need trajectory awareness, which we define as being aware of critical events that happen along multiple relevant trajectories. These critical events are often separated from the focal person both geographically and temporally, and thus do not fall in the attention circle of normal situational awareness.

Trajectory awareness is important for several reasons. First, it facilitates proactive actions in responding to unexpected interruptions. Knowing that a patient is going to be late to be transported to OR (versus already late), the charge nurse can adjust the
OR schedule to move the next case up or to fill in with an add-on case so that expensive OR time will not be wasted. Second, escalations often are caused by "waves" in OR workflow. Waving happens when OR calls for 5 patients or 5 rooms to finish at roughly the same time. Big waves can quickly and easily exhaust slack resources and create bottlenecks. Trajectory awareness can prevent waves from forming and alert relevant groups when waves do form so that they can be better prepared. Trajectory awareness can alleviate intergroup tension and conflict as well. OR is a very stressful environment and people must concentrate to get their work done. People communicate through mostly brief phone conversations and only have time to convey the most important pieces of information. Failing to share seemingly unimportant contextual information may lead to miscommunication and misunderstanding. Trajectory awareness could help build common ground as a basis for effective communication and joint problem solving.

\subsection{Importance of organizational learning}

When groups are separated by multiple boundaries, group members tend to focus their attention on local issues and fail to see the big picture of collaborating with each other. Activity Theory identifies three levels of collaborative activity as coordination, cooperation, and co-construction [1]. Most of what we observed involved coordination (routine flow of interactions) and cooperation (interactions with shared objectives), but little co-construction (re-conceptualization of ways of organizing to achieve shared objectives).

Almost all groups in the hospital practice significant amounts of book-keeping. OR nurses record the start time of every case and document reasons for delays. Post anesthesia coordinators record the in and out time for every patient and reasons for overstays. Yet the primary purpose of bookkeeping is to minimize liability rather than to improve coordination processes. Most records are in paper format and thus are not processable for systematic analysis. Across group boundaries, there is little systematic reflection, learning, and adaptation based on a holistic view of how workspaces are configured and resources are allocated. As a result, we observed continuous recurrences of certain types of escalations that resulted from a small set of bottlenecks built in the system (e.g., the shortage of escorts to transport patients). Problems were solved locally by different groups over and over again using their own pieces of evidence, rather than all the groups pooling information to identify and solve the root causes of these problems. 


\section{Computer support for multiple group coordination}

In this section, we speculate how technology might be adapted or designed to support multiple group coordination in the critical hospital setting. We draw from the literature on cognitive artifacts such as large displays (e.g. [14], [19]) and ubiquitous computing such as context-aware systems (e.g. [3], [5], [11]).

\subsection{Adapting interactive eWhiteBoards to $O R$}

We propose the idea of distributed, interactive eWhiteBoards [4] to improve trajectory awareness. In one of the OR units we studied, there is only one whiteboard outside of the nurse anesthetist office used exclusively by the anesthesia group. OR charge nurses work primarily with a printed OR schedule and the scheduling software to make changes. POHA and PACU each have their own small whiteboards that are primarily used for space and staff allocation within their own units. Consequently, every change made by OR charge nurses needs to be communicated by phone or face-to-face to all the other groups. Frustration and conflict arise when changes fail to be communicated in a timely manner.

We envision that eWhiteBoards might be placed in pre-op, the OR desk, and post-op areas, respectively. These boards can be configured to display information needed by each group. The boards can be automatically populated using information from a supporting database (an extension of the current scheduling system for instance). The three boards then communicate through the database. When charge nurses change the OR schedule, for instance, a request would be sent to the supporting database and the database automatically updates all eWhiteBoards. Thus eWhiteBoards become more than a visual display or a reference for interpersonal communication. They can prompt an innovative way of dynamic communication and help minimize the need for collaborators to intentionally communicate every action that they initiate that may interfere with other trajectories or the overall workflow.

Interactive eWhiteBoards only solve half of the problem - information still needs to be collected and entered into the system in a continuous and timely fashion. Who will be responsible for collecting and entering the data? Who will ensure the accuracy and timeliness of the data? Will the system cause even more political withholding of information and gaming of the system? We propose to address these problems by delegating the routine tasks of gathering and sharing awareness data to intelligent agents such as context- aware systems and having these tasks performed as a byproduct of ordinary work practices.

\subsection{Context-aware systems}

Context-aware technology can be use to acquire information about the physical location and social situation of a device or a person. Example systems in the operating room context may include: context-aware beds or location-aware bracelets to keep track of patient status [3], location-aware PDA to locate surgeons and anesthesiologists, and video and audio sensors to detect activities in the operating rooms [5], [11]. Standardizing and delegating routine communication tasks to context-aware systems could improve multiple group coordination in several regards.

First, this arrangement can significantly decrease the costs of gathering and sharing information. Information is collected and disseminated as a byproduct of getting work done. As a patient is transported to the preoperative holding area, for instance, the context-aware bed senses its location and automatically sends signals to update the patient's status in the supporting database. The same thing happens when a surgeon enters the hospital building. These pieces of information are then "pushed" and displayed to the groups who will be serving the patients or working with the surgeon later.

Second, this arrangement enables the decoupling of information seeking and providing [22] and may reduce the negative impact of interruptions. In face-to-face or phone communications, information seeking and providing are tightly coupled - information is provided when it is solicited. Individuals and groups constantly interrupt each other for information, and key players such as charge nurses get overloaded as the hub of information flow [17]. This arrangement will also alleviate problems associated with the political use of information and as it makes it difficult for one party to withhold information from another due to accountability concerns [25].

Revisiting the three-level model of collaboration, gathering and synthesizing information using the context-aware technology also provides a basis for groups to move from purely activity coordination to cooperation and co-construction. With critical events recorded and analyzed on a regular basis, especially critical points around which coordination breakdowns occur, systematic analyses can be conducted and used to diagnose and re-design the current way of organizing and coordinating. The goal is to hold inefficient practices or ways of coordinating accountable instead of holding human beings or groups accountable. 
A technological solution is not successful unless or until it becomes accepted and properly utilized by its targeted users. Users may feel threatened or intimidated by the technology and refuse to use it or even sabotage it [1]. Collaborative technology does not necessarily engender collaboration unless organizational norms and incentives encourage it [20]. Hence, we discuss the role and importance of fostering a collaborative culture in implementing our technical recommendations.

\subsection{Organizational culture and structure}

By a collaborative culture, we refer to key organizational elements that will positively affect workers' attitudes toward collaboration and their perception and usage of collaborative technology. Most tension and conflict that we observed was task-related. Some emerged due to different perspectives on a problematic situation or divergent goals and priorities, but in other cases there were underlying organizational politics and conflicts over power. We argue that a collaborative culture encouraging relationship building, informal communicating, and perspective taking will facilitate multiple group coordination. We illustrate the importance of fostering a collaborative culture using two examples: workplace norms and workspace configuration.

Our interview data reveal the norm in the hospitals we studied to be coordinated, somewhat cooperative, but rarely co-constructive. Teamwork or joint problem solving are manifest in carrying out ordinary work activities and solving local problems, but not in collective learning and improving ways of organizing at a higher level. Although group representatives and top management participate in a weekly joint meeting to go over important management issues, the meeting is primarily a place for conflict resolution and negotiation. When problems arise, groups try hard to hold each other accountable, rather than holding the system accountable and figuring out ways to improve it. They resolve problems that return and persist, such as the inadequate staffing of patient transportation. Had they changed their attitudes to attacking and examining the system, a straightforward analysis of supply and demand as shown in Table 1 would vividly illustrate this lack of manpower. On a daily basis, only two escorts are assigned to transport 25-30 patients back and forth between ambulatory surgery (in building $\mathrm{M}$ ) and operating rooms are located in building $\mathrm{P}$, which is a 15-minute walk. These two escorts are also responsible for transporting patients between ambulatory surgery and the operating rooms located in the same building yet one floor up. When OR "waves" form, there can be 5 to 10 patients waiting to be transported in 15 minutes, which is mission impossible.

Our observations also suggest that how workspace is configured in the operating room might contribute to and exacerbate the problems associated with multiple group coordination. Surgical, anesthesia, and nursing teams each have their own break areas, and these areas are separated from one another by doors, walls, and hallways. As a result, groups cluster in their own spaces for breaks and casual interactions while not working operating rooms. Informal communication thus reinforces rather than bridges group boundaries. Numerous studies have demonstrated the importance of informal communication and personal social networks in workplaces [13], [18], [28]. Brief, unplanned, and frequent interactions are crucial in promoting task awareness, supporting coordination of group activities, and building social relationships. We have an ongoing study that examines how workspace configuration and the position of coordination artifacts such as whiteboards affect collaboration in the OR setting.

\section{Table 1. Personnel assignment analysis}

\begin{tabular}{|l|c|c|c|c|}
\hline Roles & $\begin{array}{c}\text { Max } \\
\text { ratio }\end{array}$ & $\begin{array}{c}\text { Actual } \\
\text { ratio }\end{array}$ & Worker & Patient \\
\hline Surgeon & $1: 2$ & $1:<2$ & 20 & 25 \\
\hline Anesthesiologist & $1: 3$ & $1:<3$ & 12 & 25 \\
\hline Nurse anesthetist & $1: 1$ & $>1: 1$ & 30 & 25 \\
\hline OR nursing & $1: 1$ & $>1: 1$ & 30 & 25 \\
\hline Housekeeping & $1: 1$ & $1:>5$ & $2-5$ & 25 \\
\hline Escort & $1: 1$ & $1:>10$ & 2 & 25 \\
\hline
\end{tabular}

Note: Max ratio is the maximum number of patients that a person can care for at one time. Actual ratio is the number of workers assigned per day over the number of patients to be taken care of.

\section{Conclusion}

In this paper, we have shown that multiple trajectories that are tightly coupled and span across multiple groups in a highly complex and dynamic environment pose significant challenges for the design of collaborative technology. We conducted field observations and interviews in a local hospital to understand the sources and consequences of coordination breakdowns when groups fail to adjust to unexpected interruptions. We propose two design recommendations for the purposes of promoting trajectory awareness and learning at the organizational level. We discuss the role of a collaborative culture in facilitating the adoption and effective utilization of collaborative technology. Our goal is to provide insights and recommendations that $\mathrm{CSCW}$ designers can easily grasp and act upon. We believe our findings and recommendations have the potential to advance the 
development of collaborative technology for coordinating complex trajectories spanning multiple groups in critical environments such as hospitals.

\section{Acknowledgements}

This research is supported by NSF ITR grant IIS 0325047. The views expressed here are those of the authors and do not reflect the official policy or position of NSF. We thank Suzanne Weisband and Marina Kobayashi for their helpful comments.

\section{References}

[1] Bardram, J.E. (1997) I love the system - I just don't use it! Proc. of ACM GROUP97, 251-260.

[2] Bardram, J.E. (2000) Temporal coordination. Computer Supported Cooperative Work, 9. 157-187.

[3] Bardram, J.E. (2004) Applications of context-aware computing in hospital work - Examples and design principles. Proc. of SAC'04, 1574-1579.

[4] Berkowicz, D.A., Barnett, G.O. and Chueh, H.C. (1999) eWhiteBoards: A real time clinical scheduler. Proc. of AMIA Annual Symposium.

[5] Chen, D., Yang, J., and Wactlar, H. A Study of detecting social interaction in a nursing home environment, Proc. of IEEE HCI'05, in conjunction with ICCV'05.

[6] Choi, T., Jameson, H., and Brekke, M. L. (1991). Operations research in nurse scheduling (pp. 189-228). In National Research Council, People and Technology in the Workplace. Washington, DC: National Academy of Sciences.

[7] Flanagan, J.C. (1954) The critical incident technique. Psychological Bulletin, 51 (4). 327-358.

[8] Gaba, D., and Lee, T. (1989). Measuring the workload of the anesthesiologist. Anesthesia and Analgesia, 71, 354-361.

[9] Heath, C. and Luff, P. (1992) Collaboration and control: Crisis management and multimedia technology in London underground control rooms. Computer Supported Cooperation Work, 1(1), 69-94.

[10] Helmreich, R. and Schaefer, H-G. (1994). Team performance in the operating room (pp. 225-253). In M. S. Bogner (ed.), Human Error in Medicine. Hillsdale, NJ: Erlbaum.

[11] Kidd, C.K., Orr, R.J., Abowd, G.D., Atkeson, C.G., Essa, I.A., MacIntyre, B., Mynatt, E., Starner, T.E., and Newstetter, W. (1999) The aware home: A living laboratory for ubiquitous computing research. Proc. of CoBuild' '99.

[12] Kobayashi, M., Fussell, S.R., Xiao, Y. and Seagull, F.J. (2005) Work coordination, workflow, and workarounds in a medical context. Proc. Of CHI'05, 1561-1564

[13] Kraut, R. E., Fish, R.S., Root, R.W., \& Chalfonte, B.L. (1990) Informal communication in organizations: Form, function, and technology. In S. Oskamp \& S. Spacapan
(Eds). Human Reactions to Technology: The Claremont Symposium on Applied Social Psychology, 145-199. Beverly Hills, CA: Sage.

[14] Lasome, C.E.M. and Xiao, Y. (2001) Large public display boards: A case study of an OR board and design implications. Proc. of AMIA'01, 349-352.

[15] Miyata, Y. and Norman, D.A. (1986) Psychological issues in support of multiple activities. In Norman, D.A. and Draper, S.W. eds. User Centered System Design, Lawrence, Hillsdale, NJ, 1986, 265-284.

[16] Mortensen, M. and Hinds, P. (2001) Conflict and shared identity in geographically distributed teams. International Journal of Conflict Management, 12(3), 212-238.

[17] Moss, J. and Xiao, Y. (2002) A comparison of communication needs of charge nurses in two operating room suites. Proc. of AMIA, 543-547.

[18] Nardi, B.A., Whittaker, S., and Schwarz, H. (2002) Networkers and their activity in intensional networks. In Nardi, B. and Redmiles, D. (Eds.). Special Issue: Activity Theory and the Practice of Design. CSCW. 11:1-2. 205-42.

[19] Nemeth, C. (2003) The Master Schedule: How Cognitive Artifacts Affect Distributed Cognition in Acute Care, The Union Institute and University, Cincinnati, $\mathrm{OH}$.

[20] Orlikowski, W.J. (1992) Learning from notes: Organizational issues in groupware implementation. Proc. of CSCW'92, 362-369.

[21] QSR International. (1999) QSR NUD*IST Vivo. Doncaster, Australia.

[22] Reddy, M. and Dourish, P. (2002) A finger on the pulse: Temporal rhythms and information seeking in medical work. Proc. of CSCW'02, 344-353.

[23] Seagull, F.J., Plasters, C., Xiao, Y. and Mackenzie, C.F. (2003) Collaborative management of complex coordination systems: Operating room schedule coordination. Proc. of HFES'03.

[24] Strauss, A., Fagerhaugh, B., Suczek, B., and Weiner, C. (1985) The Social Organization of Medical Work. Chicago: University of Chicago Press.

[25] Symon, G., Long, K. and Ellis, J. (1996) The coordination of work activities: Cooperation and conflict in a hospital context. Computer Supported Cooperative Work, 3, 1-31.

[26] Thompson, J.D. (1967) Organizations in Action: Social Science Bases of Administrative Theory. McGraw-Hill, New York.

[27] Weinger, M. and Englund, C. (1990) Ergonomic and human factors affecting anesthetic vigilance and monitoring performance in the operating room environment. Anesthesiology, 73, 995-1021.

[28] Whittaker, S., Frohlich, D. and Daly-Jones, O. (1994) Informal workplace communication: What is it like and how might we support it? Proc. of HFCS'94, 131-137.

[29] Xiao, Y., Seagull, F.J., Faraj, S. and Mackenzie, C.F. (2003) Coordinating practices for patient safety: Knowledge, cultural, and supporting artifact requirements. Proc. of Ergonomic'03. 